\title{
BMJ Open The diagnostic performance evaluation of the SD BIOLINE HIV/syphilis Duo rapid test in southern Ethiopia: a cross-sectional study
}

\author{
Techalew Shimelis, Endale Tadesse
}

To cite: Shimelis T, Tadesse E. The diagnostic performance evaluation of the SD BIOLINE HIV/syphilis Duo rapid test in southern Ethiopia: a cross-sectional study. BMJ Open 2015;5: e007371. doi:10.1136/ bmjopen-2014-007371

- Prepublication history for this paper is available online. To view these files please visit the journal online (http://dx.doi.org/10.1136/ bmjopen-2014-007371).

Received 5 December 2014 Revised 23 February 2015 Accepted 20 March 2015

CrossMark

Department of Medical Laboratory Science, Hawassa University, Hawassa, Ethiopia

Correspondence to Techalew Shimelis; techalew03@yahoo.com

\section{ABSTRACT}

Objective: To determine the diagnostic performance of the SD BIOLINE HIV/syphilis Duo rapid test.

Design: A hospital-based cross-sectional study.

Setting: This evaluation was conducted at one of the largest hospitals in southern Ethiopia.

Participants: Serum samples obtained from clients attending the antiretroviral therapy and voluntary counselling and testing centres were used. Sera were originally collected for the purpose of investigating syphilis epidemiology. The performance of the test to detect HIV was evaluated using 400 sera (200 HIV positives and $200 \mathrm{HIV}$ negatives). Also, its performance to detect syphilis was evaluated using 85 syphilis positive and 100 syphilis negative serum samples. Individuals $<15$ years of age or syphilis treated or those with $\leq 50$ cells/uL CD4 cell count were originally excluded.

Outcome measures: HIV screening was carried out according to the national rapid diagnostic testing (RDT) algorithm: Shenghai Kehua Bioengineering (KHB) test kit as a screening test, followed by the HIV1/2 STAT-PAK assay if positive. Where the result of the STAT-PAK is discordant with KHB, Unigold HIV is used as a tiebreaker to determine the result. We also used ELISA to resolve discordant HIV results. Syphilis serostatus was determined using the Treponema pallidum haemagglutination assay (TPHA).

Results: The respective sensitivity, specificity, positive predictive value and negative predictive value of the $S D$ BIOLINE HIV/syphilis Duo test were 100, 99.5, 99.5 and $100 \%$ for HIV and $97.6,96,95.4$ and $98 \%$ for syphilis testing, respectively. In reference to TPHA, the test kit reported 4 false positives and 2 false negative results for syphilis. The $\kappa$ values were 0.99 for HIV testing and 0.94 for syphilis testing.

Conclusions: The excellent performance of the SD BIOLINE HIV/syphilis Duo test to detect HIV as well as syphilis facilitates the integration of syphilis testing and treatment to the already established HIV prevention programme, ultimately contributing to the dual HIV and syphilis elimination goal.

\section{INTRODUCTION}

Sexually transmitted infections (STI) are one of the major public health problems

\section{Strengths and limitations of this study}

- Whether the test shows similar performance in field conditions where whole blood is used for HIV testing requires further investigation.

- Discordant results between two tests for HIV may better be resolved using advanced techniques such as Western Blot and PCR tests.

- False-negative results in recent syphilis infection and false-positive results in the absence of disease activity limit interpretation of treponemal test results.

worldwide, especially in developing countries where access to adequate diagnostic and treatment facilities are very limited or nonexistent. ${ }^{1}$ Syphilis is among the oldest STIs known to mankind, caused by the bacterium Treponema pallidum. ${ }^{2}$ It has been estimated that annually about 12 million new infections occur worldwide, of which almost two-thirds are in sub-Saharan Africa and South and Southeast Asia. ${ }^{3}{ }^{4}$ It was estimated in 2008 that 1.4 million women globally had syphilis, and up to $80 \%$ of untreated mothers develop adverse pregnancy outcomes including stillbirth, premature delivery, low birth weight and neonatal death. ${ }^{5}$

In Ethiopia, syphilis has been reported for centuries, yet its health and socioeconomic impact is not well known due to several factors like social stigma and associated under-reporting, asymptomatic nature of the infection and lack of diagnostic facilities. ${ }^{6}$ Small-scale studies reported syphilis prevalence that ranges from $1 \%$ to $10.9 \%$ in diverse risk groups such as pregnant women, blood donors, street dwellers and elderly people. $^{7-10}$ Moreover, the antenatal care (ANC)-based sentinel surveillances showed increasing prevalence of the disease from $1.8 \%$ in the year 2003 to $2.7 \%$ in 2005 and then stabilised at $2.3 \%$ in 2007 and $2009 .{ }^{11-14}$ 
Mainly as a result of shared transmission routes, syphilis-HIV coinfection is a common public health problem. ${ }^{15}$ We previously reported an eightfold higher rate of syphilis in HIV-infected patients compared to HIV-uninfected individuals in Addis Ababa. ${ }^{16}$ It was also shown from previous surveillances in Ethiopia that dual infection among ANC attendees rose from $4.1 \%$ in the year 2003 to $4.9 \%$ in 2005 and $5.3 \%$ in 2007, but dropped back to $3.9 \%$ in $2009 .{ }^{11-14}$ HIV infection has been declining as a result of comprehensive and expanded responses, which make the HIV screening service more accessible for pregnant women and other subpopulations. Expanding HIV testing service has become possible with developing testing guidelines that direct the use of rapid diagnostic test (RDT) algorithm. In contrast, although policies on syphilis screening of pregnant women have been in place, lack of suitable diagnostic methods challenge the implementation of syphilis testing in peripheral settings. Thus, most pregnant women are unaware of their infection status to seek treatment and may pass syphilis on to their babies. ${ }^{17}$

To date, several reagent companies have developed RDTs detecting syphilis. However, the SD BIOLINE HIV/ syphilis Duo test has emerged with a major advance, enabling the dual detection of syphilis and HIV infections. This simple test addresses the applicability challenges associated with the traditional syphilis tests (eg, rapid plasma reagin, venereal disease research laboratory), requiring a laboratory set-up, electricity supply and qualified laboratory personnel. Thus, it facilitates the expansion of screening service in pregnant women or other subpopulations in a variety of urban and rural settings. Since RDTs are performed at or near the site of care and results are obtained without having to wait days or even hours, it allows clinicians and patients to make a quick clinical decision. ${ }^{18}$ The excellent diagnostic performance of the SD BIOLINE HIV/syphilis Duo test, as claimed by the manufacturer and supported with couple of emerging reports, ${ }^{19}{ }^{20}$ may make it an attractive possible alternative to the traditional methods. However, since the test performance for such products is influenced by factors like infection prevalence, studied population and genetic diversity of causative agents, the need for extensive evaluations in various geographical regions is to be emphasised. Limited evidence exists regarding the diagnostic usefulness of this product in Ethiopian contexts where both infections are prevalent. Therefore, this study aimed at evaluating the performance of the SD BIOLINE HIV/syphilis Duo test kit so that its diagnostic significance would be elucidated.

\section{METHODS}

\section{Setting and samples}

This cross-sectional study was conducted at Hawassa University Hospital, southern Ethiopia. The hospital routinely provides HIV testing and counselling service for clients at the voluntary counselling and testing (VCT)
Center. Those found to be HIV infected enrol and monitor their disease status at the antiretroviral therapy (ART) clinic of the hospital. Sera collected 6 months prior to the current study for the purpose of investigating epidemiology of syphilis in a consecutive series ART and VCT clients were used. Individuals under 15 years of age or syphilis treated or those HIV-infected patients with $\leq 50$ cells $/ \mu \mathrm{L}$ CD4 T-cell count were originally excluded. The test kit's performance to detect HIV was evaluated using 400 sera (200 HIV positive and $200 \mathrm{HIV}$ negative) selected randomly from 1000 ART and 500 VCT samples. However, an attempt was made to include all HIV-syphilis coinfected serum samples. In total, 185 sera, consisting of 85 syphilis positive and 100 negative samples, were analysed to determine the performance of the test to detect syphilis.

\section{HIV and syphilis testing}

Routine HIV testing in Ethiopia is performed uniformly on the basis of the established national rapid testing algorithm consisting of three RDTs in series. It uses the KHB test kit (Shenghai Kehua Bioengineering Co. Ltd., China) as a screening test, followed by the HIV1/2 STAT-PAK assay (Chembio Diagnostic Systems Inc., USA) if positive. A negative KHB test result is reported without the need for further confirmation. Where the result of STAT-PAK is discordant with KHB, a third test, Unigold HIV (Trinity Biotech Plc., Ireland), is used as a tiebreaker to determine the result. Thus, sera characterised with this HIV testing algorithm were analysed by the SD BIOLINE HIV/syphilis Duo test (Standard Diagnostic Inc., Korea). In cases where the result of the SD BIOLINE HIV/syphilis Duo test is discordant with the specified HIV testing algorithm, we used an ELISA (Vironostika HIV1/2 Uniform II plus O; Biomerieux BV, Boxtel, the Netherlands) and its result is conclusive. The flow diagram illustrating the HIV testing structure is shown in figure 1 . The performance of the new kit to detect syphilis was assessed in comparison to the syphilis serostatus as determined by the modified $T$. pallidum haemagglutination assay (TPHA).

The SD BIOLINE HIV/syphilis Duo test kit is a solid phase immunechromatographic assay for qualitative detection of antibodies to all isotopes (IgG, IgM, IgA) specific to HIV-1/2 and/or Treponema palladium in human serum, plasma and whole blood. The test procedure is to add $10 \mu \mathrm{L}$ of serum to a sample well, and then to add three drops of assay diluent to the same well. The result is read at $15-20 \mathrm{~min}$. A positive test result is indicated when the control line $(\mathrm{C})$ and test line ('SYP' or 'HIV' or both) are visible, and a negative result is indicated when only the control line $(\mathrm{C})$ is visible. The test is invalid when the control line is invisible. Four Laboratory Technologists (Bachelor of Science Degree holders in Medical Laboratory Technology) with at least a year's work experience carried out the HIV rapid test algorithm, SD BIOLINE HIV/syphilis Duo test, ELISA and 
Figure 1 A flow diagram illustrating the HIV testing structure, southern Ethiopia, 2014. (-), negative; (+), positive; ELISA n, number.

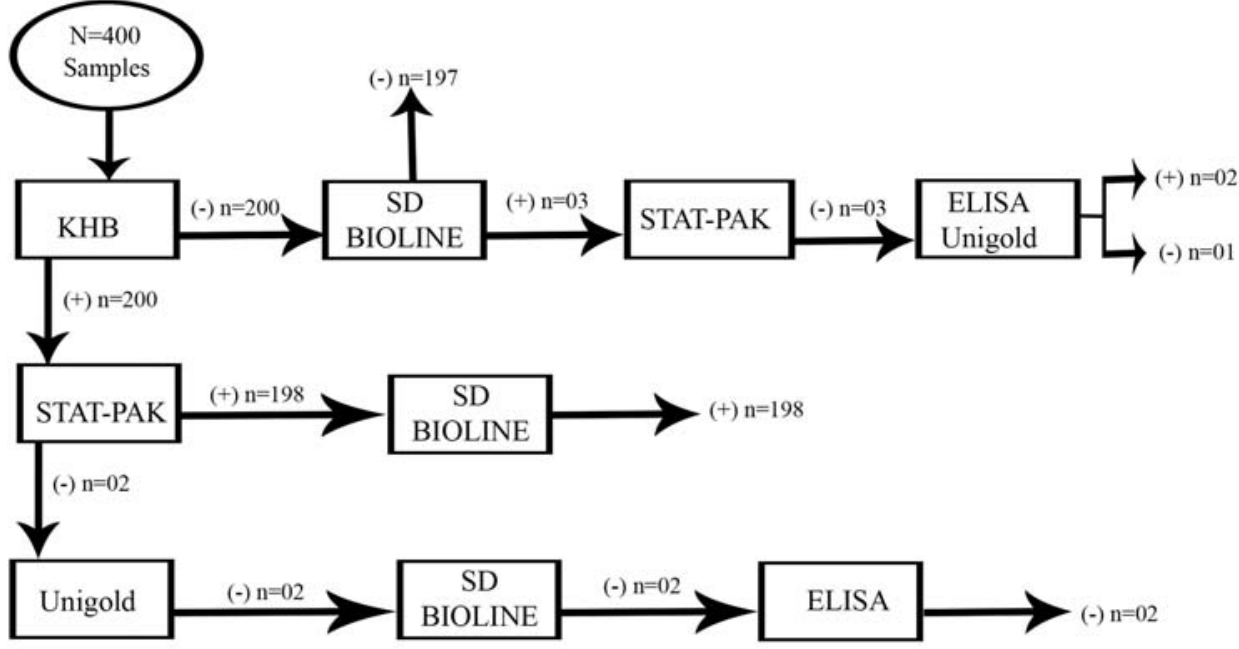

$(-)$, Negative; (+), Positive; ELISA, enzyme linked immunosorbent assay; n, number
TPHA as specified by the manufacturer. Investigators were blinded to each other's test results.

\section{Data analysis}

Data entry and analysis were performed using STATA V.10. Test parameters of the test kit including sensitivity, specificity, positive predictive value and negative predictive value, each with $95 \% \mathrm{CI}$, were determined. The $\mathrm{\kappa}$ statistic was calculated to show the degree of agreement between the RDT and the reference method.

\section{Ethical clearance}

This study was approved by the Institutional Review Board of the College of Medicine and Health Sciences, Hawassa University. Informed written consent was originally obtained from the study participants to use the leftover sera from the previous study for the current investigation. Any information obtained during the study is kept with utmost confidentiality, and those found infected with syphilis were treated.

\section{RESULTS}

Sera collected from a total of 400 individuals were analysed during June-August 2014. The male to female ratio was $0.78: 1$ in HIV-infected participants and 1:1 in those with no HIV infection. The mean age of HIV-infected participants was 33.9 (range 15-75; SD 9.5), and that of HIV-uninfected participants was 35.3 (range 16-75; SD 14.3). The median CD4+T-cell count for HIV infected clients was 402 cells $/ \mu \mathrm{L}$ (range $51-1213$ cells $/ \mu \mathrm{L}$ ). As shown in figure 1, among 200 samples that tested HIV negative by KHB, two were tested positive by Unigold or ELISA. Furthermore, two other samples tested positive by KHB were found negative by other three tests (STAT-PAK/Unigold/ELISA). Thus, in reference to ELISA, KHB produced false negative results in two samples, and of course those samples were false negative by STAT-PAK as well. While KHB reported a false positive result in two other samples, those participants with discordant HIV results had a CD4+T-cell count $\geq 374$ cells $/ \mu \mathrm{L}$.

A total of 185 TPHA characterised serum samples were analysed for this kit's performance evaluation, 85 of which were positive for syphilis. Sixty-seven of the syphilis positives and 59 of the syphilis negative samples were HIV positive (table 1 ).

The results of the SD BIOLINE HIV/syphilis Duo test kit are presented in table 2. The test has correctly determined HIV serostatus in all samples except in a sample that was found false positive. The SD BIOLINE HIV/syphilis Duo test agreed with STAT-PAK/Unigold/ ELISA results in instances where KHB generated false positive results. Moreover, the SD BIOLINE HIV/ syphilis Duo test reported concordant results in 83 of 85 syphilis positives and in 96 of 100 syphilis negative samples. Thus, false positive and false negative results for syphilis were reported in four and two samples, respectively.

The respective sensitivity, specificity, PPV, NPV and accuracy of the SD BIOLINE HIV/syphilis Duo test were calculated to be $100,99.5,99.5,100$ and $99.8 \%$ for detection of HIV infection. Moreover, the test kit detected syphilis with sensitivity, specificity, PPV, NPV and accuracy of 97.6, 96, 95.4, 98 and 96.6\%,

Table 1 Distribution of the syphilis test result by HIV status as determined by the reference method, southern Ethiopia, 2014

\begin{tabular}{lllll}
\hline \multirow{2}{*}{ Syphilis-reference } & & \multicolumn{3}{c}{ HIV-reference } \\
\cline { 3 - 5 } & & Positive & Negative & Total \\
\hline TPHA & Positive & 67 & 18 & 85 \\
& Negative & 59 & 41 & 100 \\
& Total & 126 & 59 & 185
\end{tabular}

Syphilis reference method, TPHA; HIV reference method, KHB/ STAT-PAK/Unigold algorithm+ELISA.

KHB, Shenghai Kehua Bioengineering; TPHA, Treponema pallidum haemagglutination assay. 
Table 2 Assay results of the SD BIOLINE HIV/syphilis Duo test for dual detection of HIV and syphilis, southern Ethiopia, 2014

\begin{tabular}{|c|c|c|c|c|c|}
\hline \multirow[b]{2}{*}{ RDT } & \multirow[b]{2}{*}{ Infection } & \multirow[b]{2}{*}{ RDT result } & \multicolumn{3}{|c|}{ Reference method* } \\
\hline & & & Positive & Negative & Total \\
\hline \multirow[t]{6}{*}{ SD HIV/syphilis Duo } & HIV & Positive & 200 & 1 & 201 \\
\hline & & Negative & 0 & 199 & 199 \\
\hline & & Total & 200 & 200 & 400 \\
\hline & Syphilis & Positive & 83 & 4 & 87 \\
\hline & & Negative & 2 & 96 & 98 \\
\hline & & Total & 85 & 100 & 185 \\
\hline
\end{tabular}

*Syphilis reference method, TPHA; HIV reference method, KHB/STAT-PAK/Unigold algorithm+ELISA.

KHB, Shenghai Kehua Bioengineering; RDT, rapid diagnostic test; TPHA, Treponema pallidum haemagglutination assay.

respectively (table 3). A strong degree of agreement between the test and reference methods were shown, in which $\kappa$ values were 0.99 for HIV testing and 0.94 for syphilis testing.

\section{DISCUSSION}

We evaluated the diagnostic performance of the SD BIOLINE HIV/syphilis Duo test, which is designed to dually detect HIV and syphilis. The sensitivity and specificity of the kit to detect HIV infection were $100 \%$ and $99.5 \%$, respectively. Similar performance results were reported in two studies that evaluated the same diagnostic product using samples from Africa. ${ }^{19} 20$ The capability of the SD BIOLINE HIV/syphilis Duo test to detect those HIV-infected cases missed by the currently used screening test (KHB) highlights the usefulness of the new product as an HIV screening test. Thereby, the consequences of false negative results in relation to HIV transmission in various subpopulations, especially in pregnant women, blood donors, married couples and VCT clients, could be minimised. Even though the observed KHB false positive results may be traced with series testing employed in the current HIV rapid testing algorithm, providing HIV screening with an SD BIOLINE HIV/syphilis Duo test could minimise the occurrence of such cases and save time, money and labour. However, the HIV false positive result by the SD BIOLINE HIV/syphilis Duo test in one sample may indicate the importance of confirming positive results with more specific tests.

The SD BIOLINE HIV/syphilis Duo test detected syphilis with sensitivity and specificity of 97.6 and $96 \%$, respectively. A comparable performance was reported by Bristow et $a l^{19}$ for the same test product (sensitivity and specificity, 99.7\%). The SD BIOLINE HIV/syphilis Duo is simple to test and can be performed by a variety of healthcare workers, increasing the number of people that can be tested for HIV and syphilis at the point of care. Results from the test are available within $20 \mathrm{~min}$, allowing clients to be diagnosed and to receive timely management. As the test does not require a laboratory set-up, equipment or electricity, it fits into resourcelimited settings and can function in rural areas where most of our people reside. The small volume of blood required and variety of sample (whole blood, serum or plasma) that can be used for the test as well as a convenient test procedure and easy interpretation of results make the method attractive and user-friendly. However, since the predictive value of a test depends on the prevalence of a particular infection, the test may also demonstrate different performance elsewhere if the prevalence is lower.

The limited availability of diagnostic options in most resource-constrained parts of the world hinder the scale up of syphilis intervention strategies. Thus, the infection has remained a public health problem for centuries despite the availability of effective treatment that could diminish its clinical and socioeconomic impact. ${ }^{21}$ Further, the spread of syphilis may adversely influence HIV transmission by raising the viral load as well as presenting site for HIV entry and shading. ${ }^{15}$ Now, the SD BIOLINE HIV/syphilis Duo test has emerged with several merits to implement the dual screening of HIV and syphilis. The advent of such good performing diagnostic products enhances the accessibility of quality screening service in resource-constrained countries. Thus, it enforces a syphilis screening programme, which has been provided irregularly for limited subpopulations including pregnant women, blood donors and patients with clinical indications. By detecting syphilis early at the

Table 3 Diagnostic performance characteristics of the SD BIOLINE HIV/syphilis Duo test kit, southern Ethiopia, 2014

\begin{tabular}{llllll}
\hline \multirow{2}{*}{ RDT } & Infection & $\begin{array}{l}\text { \% Sensitivity } \\
\mathbf{9 5 \%} \mathbf{C l}\end{array}$ & $\begin{array}{l}\text { \% Specificity } \\
\mathbf{9 5 \%} \mathbf{C l}\end{array}$ & $\begin{array}{l}\text { \% PPV } \\
\mathbf{9 5 \%} \mathbf{~ C l}\end{array}$ & $\begin{array}{l}\text { \% NPV } \\
\mathbf{9 5 \%} \mathbf{~ C l}\end{array}$ \\
\hline SD HIV/syphilis Duo & HIV & $100(98.5$ to 100$)$ & $99.5(97.6$ to 100$)$ & $99.5(97.6$ to 100$)$ & $100(98.5$ to 100$)$ \\
& Syphilis & $97.6(92.4$ to 99.6$)$ & $96(90.6$ to 98.7$)$ & $95.4(89.3$ to 98.5$)$ & 98 (93.4 to 99.7) \\
\hline
\end{tabular}

Syphilis reference method, TPHA; HIV reference method, KHB/STAT-PAK/Unigold algorithm+ELISA.

KHB, Shenghai Kehua Bioengineering; NPV, negative predictive value; PPV, positive predictive value; RDT, rapid diagnostic test. 
time of HIV screening, its clinical consequences in people living with HIV could be minimised. With this diagnostic product, the integration and expansion of HIV and syphilis intervention programmes would be facilitated so that the strategy for dual elimination of mother to child transmission of HIV and syphilis might be successful.

However, this study was limited to evaluating the test kit's performance using a serum sample. As to whether the test shows similar performance in field conditions in which whole blood is used for HIV testing requires further investigation. Moreover, no effort was made to further characterise HIV false positive and false negative samples using techniques such as Western Blot and PCR to adequately explain those results. Assessing the performance of the new test in comparison with the HIV rapid testing algorithm may not generate sufficient information as to how the test could combine with other RDTs and form a new testing algorithm. However, the evidence from this study helps decision-makers to be informed regarding the significance of considering the new test in our HIV testing algorithm protocol. Although TPHA is a confirmatory test in our context, the limitations of a possible false negative result in recent syphilis infection and a false-positive result in the absence of disease activity hinder the interpretation of treponemal test results. Whether the SD BIOLINE HIV/syphilis Duo test, which has excellent agreement with TPHA, suffers from the same limitations needs further investigation.

In summary, the excellent sensitivity and specificity of the SD BIOLINE HIV/syphilis Duo test for dual detection of HIV and syphilis indicate the diagnostic usefulness of the test to screen various subpopulations. With this test, the integration of syphilis to the already established HIV prevention programme would be possible. Thus, the existing weaker syphilis intervention efforts could be strengthened, which ultimately diminish the impact of the disease. Further study of HIV rapid testing algorithm and consideration of a test with such major breakthrough presents an opportunity to all those who get HIV testing be automatically screened for syphilis as well. Such a testing strategy would be crucial to realise the global efforts of dual elimination of HIV and syphilis in pregnant women or other at-risk subpopulations.

Acknowledgements The authors would like to thank the Hawassa University for financial support. They are also most grateful to the Standard Diagnostics Inc. for providing kits free of charge.

Contributors TS designed the study and carried out the laboratory work; TS and ET performed the statistical analyses and interpretation and contributed to the write-up. Both authors read and approved the final version of the manuscript.

Funding The study was supported with a small grant obtained from Hawassa University.

Competing interests None declared.

Patient consent Obtained.
Ethics approval The study was approved by the Institutional Review Board of the College of Medicine and Health Sciences, Hawassa University.

Provenance and peer review Not commissioned; externally peer reviewed.

Data sharing statement No additional data are available.

Open Access This is an Open Access article distributed in accordance with the Creative Commons Attribution Non Commercial (CC BY-NC 4.0) license, which permits others to distribute, remix, adapt, build upon this work noncommercially, and license their derivative works on different terms, provided the original work is properly cited and the use is non-commercial. See: http:// creativecommons.org/licenses/by-nc/4.0/

\section{REFERENCES}

1. World Health Organization (WHO). Guidelines for the management of sexually transmitted infections. Revised Version. Geneva, Switzerland: WHO, 2003.

2. Ho LE, Lukehart AS. Syphilis: using modern approaches to understand an old disease. J Clin Invest 2011;121:4584-92.

3. Lynn WA, Lightman S. Syphilis and HIV: a dangerous combination. Lancet Infect Dis 2004;4:456-66.

4. World Health Organization. Global Elimination of Syphilis: rationale and strategy for action, 2007.

5. Berman SM. Maternal syphilis: pathophysiology and treatment. Bull World Health Organ 2004;82:433-8.

6. Kassa A, Shume A, Kloos H. Sexually transmitted infections. In: Berhane Y, Hailemariam D, Kloos H, eds. Epidemiology and ecology of health and diseases in Ethiopia. 1st edn. Addis Ababa: Shama Books, 2006:435-45.

7. Andargachew M, Afework K, Belay T, et al. Sero-prevalence of Syphilis and HIV-1 during pregnancy in a teaching hospital in northwest Ethiopia. Japan J Infect Dis 2007;60:193-5.

8. Tessema B, Yismaw G, Kassu A, et al. Seroprevalence of HIV, HBV, HCV and syphilis infections among blood donors at Gondar University Teaching Hospital, Northwest Ethiopia: declining trends over a period of five years. BMC Infect Dis 2010;10:111.

9. Kassu A, Mekonnen A, Bekele A, et al. HIV and syphilis infection among elderly people in northwest Ethiopia. Japan Infect Dis 2004;57:264-7.

10. Moges F, Kebede Y, Kassu A, et al. Seroprevalence of HIV, HBV infections and syphilis among street dwellers in Gonder city, Northwest Ethiopia. Ethiop J Health Dev 2006;20:160-5.

11. Ministry of Health, Disease Prevention and Control Department AIDS in Ethiopia. Technical document for the fifth report. 2004.

12. Federal Ministry of Health. National HIVIAIDS Prevention and Control Office. AIDS in Ethiopia. Sixth report. 2006.

13. Federal Ministry of Health/Ethiopian Health and Nutrition Research Institute. Report on the 2007 round antenatal care sentinel HIV surveillance in Ethiopia. 2010.

14. Ethiopian Health and Nutrition Research Institute. Report on the 2009 round antenatal care sentinel HIV surveillance in Ethiopia. 2011.

15. Kassutto S, Sax P. HIV and syphilis co-infection: Trends and interactions. AIDS Clinic Care 2003;15:9-18.

16. Eticha BT, Sisay Z, Alemayehu A, et al. Seroprevalence of syphilis among HIV-infected individuals in Addis Ababa, Ethiopia: a hospital-based cross-sectional study. BMJ Open 2013;3:e002293.

17. Strategic plan II. For intensifying multisectoral HIV and AIDS response in Ethiopia. 2010/2011-2014-2015. Addis Ababa, Ethiopia: Federal HIV/AIDS Prevention and Control Office/Ministry of Health, 2010.

18. Drain PK, Hyle EP, Noubary F, et al. Diagnostic point-of-care tests in resource-limited settings. Lancet Infect Dis 2014;14:239-49.

19. Bristow CC, Adu-Sarkodie Y, Ondondo O, et al. Multisite laboratory evaluation of a dual human immunodeficiency virus (HIV)/syphilis point-of-care rapid test for simultaneous detection of HIV and syphilis infection. OFID 2014;1:ofu015.

20. Omoding D, Katawera V, Siedner M, et al. Evaluation of the SD Bioline HIV/Syphilis Duo assay at a rural health center in Southwestern Uganda. BMC Res Notes 2014;7:746.

21. Kamb ML, Newman LM, Riley PL, et al. A road map for the global elimination of congenital syphilis. Obstet Gynecol Int 2010:12798. doi:10.1155/2010/312798. 